\title{
Co-Inoculation of Pleurotus ostreatus with Beneficial Bacteria Enhanced Substrate Utilization and Mushroom Production: An Implication for the Development of a Novel Bio-Fertilizer
}

\author{
Dhanushika P.T.N. and Jayasinghearachchi H.S.* \\ Department of Botany, University of Sri Jayewardenepura, Sri Lanka \\ *jayasinghe148@gmail.com
}

\begin{abstract}
The oyster mushroom (Pleurotus ostreatus) is one of the most widely cultivated edible mushrooms around the world. Mycelial colonization by beneficial bacteria has been shown to promote the growth of $P$. ostreatus and the other cultivated mushrooms. Therefore, present study was conducted to investigate the effect of beneficial bacteria which was isolated from soil, on growth, mushroom yield, primordia initiation of $P$. ostreatus, reducing sugar release, secretion of lignolytic enzymes such as laccase and manganese peroxidase aiming at developing a novel bacterial inoculum for $P$. ostreatus cultivation.

Minimal salt medium (MSM) supplemented with cellulose and/or starch as a sole source of carbon and energy was used to isolate bacteria from soil whereas Ashby's nitrogen free mannitol medium was used for the isolation of free-living nitrogen fixing bacteria. Pure cultures of bacterial isolates were obtained after several rounds of streaking on solid plates of the same medium, and co-cultured them with $P$. ostreatus to screen the isolates which did not cause inhibitory effects on mycelial growth. The beneficial isolates were identified based on Gram staining morphology and biochemical tests. While in vitro mycelial colonization studies were performed in liquid medium, the in vivo studies were conducted in pots filled with sterilized lignocellulosic solid substrate to evaluate beneficial effects of isolates on growth, mushroom yield, nitrogen content, primordia initiation of $P$. ostreatus and laccase and manganese peroxidase activities. Pots which did not receive any bacterial inoculum were treated as control.
\end{abstract}

Heavy mycelial colonization by beneficial isolates was observed in liquid cultures. Mushroom yield was enhanced by two-fold in pots treated with microbial inoculum. While $30 \%$ and $50 \%$ increase in laccase and manganese peroxidase activities were observed in pots treated with bacterial inoculum than that of control pots, about $42.73 \%$ increment in nitrogen content of the dried mushroom samples was observed in treated pots. This could be possibly due to the presence of diazotrophs, cellulolytic and amylolytic isolates in the inoculum. Significant increase $(\mathrm{p}<0.05)$ in median values of primordia formed in pots treated with bacterial inoculum was also observed. The results of this study, therefore clearly indicate the importance of the application of such microbial inoculum as a bio-fertilizer in mushroom cultivation.

Keywords: Pleurotus ostreatus, Laccase, Bio-fertilizer, Co-inoculation, Mycelial colonization

Proceedings of the International Forestry and Environment Symposium 2016, Department of Forestry and Environmental Science, University of Sri Jayewardenepura, Sri Lanka. 